\title{
SYNTHESIS OF SIMPLE FEED-FORWARD NETWORKS: A FIRST-ORDER EXAMPLE
}

\author{
Sridevi V. Sarma, Munther A. Dahleh* \\ Massachusetts Institute of Technology \\ Laboratory of Information and Decision Systems \\ sree@mit.edu, dahleh@mit.edu
}

\begin{abstract}
Stability analysis of networks has been the focus of much research over the past decade. Presently, researchers are investigating performance and synthesis of controllers and channel coding schemes in networks. Such design problems are difficult in general, as there is a strong interplay between control objectives and communication constraints, which forces the synthesis of controllers and channel encoders to be done simultaneously. Current approaches typically fix one, while the other is designed to meet some objective. In this paper, we consider a simple network, in which the plant and controller are local to each other, but are together driven by a remote reference signal that is transmitted through a noisy discrete channel. We first construct a model matching performance metric that captures the tradeoffs between coding the reference command to achieve more accuracy at the remote site and designing a controller to meet performance. We then simultaneously synthesize the controller and encoder block lengths that meet the specified objective for a first-order plant and model case. Finally, we illustrate performance sensitivity to the poles of the plant and model, and to the channel noise.
\end{abstract}

\section{INTRODUCTION}

Due to the information-rich world we live in today, control in distributed, asynchronous, networked environments is in demand. Communication links that have rate limitations, delays, and that are noisy, are now heavily connected to control systems, and the interactions between the two cannot be ignored.

Much research in this area has focused on the simple feedback network shown in Figure 1 . We call a system which has exactly one plant $(\mathrm{G})$, one controller $(\mathrm{K})$, and one communication link, encoder-channel-decoder (E-C-D), a simple network. Investigators have gained tremendous insight into the interplay between communication and control by conducting stability analysis for the feedback case. They derived conditions on the channel rate required for various notions of asymptotic stability $[1,3,4,5,7,9,12,13,16$, 17], and as expected, the conditions on the channel rate heavily depend on the dynamics of the plant.

More recently, people have conducted performance analysis and have dabbled with the problem of synthesizing the controller and/or encoding scheme to achieve control performance objectives [11, 2, 14, 15]. Martins \& Dahleh conducted performance analysis and developed criteria that show the explicit interplay between disturbance rejection and channel capacity for the feedback set up, when a stochastic disturbance drives the system [11]. Sarma etal. synthesized

*This research has been supported by AFOSR: 6892167.

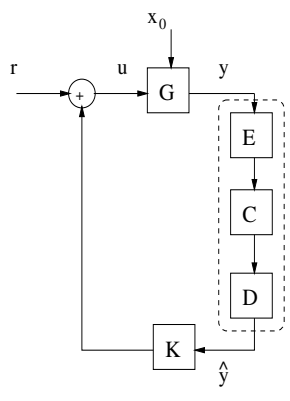

Fig. 1. Simple Feedback Network

a time-varying encoding scheme that minimized the rate required for input-output stability, when reference signals from specific deterministic classes of signals drive the system [15]. Borkar \& Mitter showed that the synthesis of the controller and encoding scheme is possible and can be separated under certain conditions, for an extended LQG problem, where the simple feedback network has additive Gaussian disturbance at the plant output [2].

In this paper, we consider the simple feed-forward network, shown in Figure 2, in which a plant and controller are both remote and separated from the reference command by a discrete communication channel. There is much work that focuses on the reconstruction of the reference command at the remote site (see [18] and references therein). However, here we are interested in driving a remote system with the reconstructed command. Most teleoperation systems, which often involve hazardous and unstructured tasks, can be addressed under this framework. Examples of such tasks include nuclear reactors, space applications, medical operations, and deep-sea and MARS explorations [10].

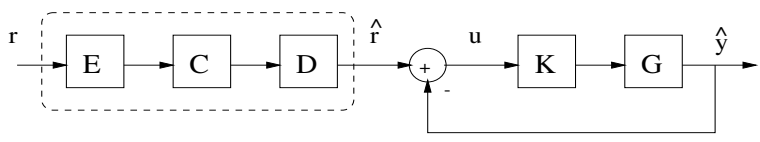

Fig. 2. Simple Feed-Forward Network

We first construct a performance metric that illustrates the tradeoffs between sending the remote control system an accurate reference command, and designing a controller such that the remote system matches a given ideal transfer function. The longer one spends coding the input signal before it enters the channel, the more accurate the signal is 
that drives the remote control system. However, delays in receiving commands at the remote site negatively affect performance. We then simultaneously synthesize the controller and encoder lengths that meet specified model matching objectives in the case where the encoder generates block codes [8], and the plant and ideal model are both first-order SISO systems. In general, synthesis of each cannot be done separately due to the tight interplay between the communication link and control system. Finally, we illustrate performance sensitivity to the poles of the plant and model, and to the channel noise.

\section{PROBLEM FORMULATION}

\subsection{Set Up}

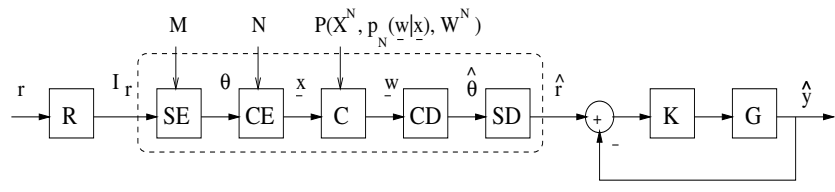

Fig. 3. Problem Set Up

We consider the set up shown in Figure 3, where

- $r \in \mathcal{C}_{r}=\left\{r_{1}, r_{2}, \ldots, r_{L}\right\}$ is a given finite set of reference signals that may be transmitted,

- $R: \mathcal{C}_{r} \rightarrow I_{r}$, where $I_{r}=\{1,2, \ldots, L\}$ is an index set, where index $j$ represents $r_{j}$, for $j=1,2, \ldots, L$,

- $S E:\{1,2, \ldots, L\} \rightarrow\{0,1\}^{\log _{2}(M)}$ is a source encoder that compresses information about the input signals $(M \leq L)$,

- $C E:\{0,1\}^{\log _{2}(M)} \rightarrow\{0,1, \ldots, k\}^{N}$ is a block channel encoder [8],

- $C$ : is a discrete memoryless channel with input domain $X^{N} \in\{0,1, \ldots, k\}^{N}$, range $W^{N} \in\{0,1, \ldots, j\}^{N}$, and corresponding conditional probability distribution $P_{N}(\underline{w} \mid \underline{x})$,

- $C D$ : is a channel decoder that maps $W^{N} \rightarrow$ $\{0,1\}^{\log _{2}(M)}$ to minimize the probability of decoding error, $P(\theta \neq \hat{\theta})$,

- $S D:\{0,1\}^{\log _{2}(M)} \rightarrow \mathcal{C}_{r}$ maps the estimate $\hat{\theta}$ to one of the $L$ reference signals ${ }^{1}$,

- $K$ is a causal, discrete-time, SISO controller, and

- $G$ is an unstable causal, discrete-time, SISO plant.

\footnotetext{
${ }^{1}$ We assume that the source decoder carries in its memory a bank of al possible reference signals in $\mathcal{C}_{r}$, and that it activates one of them when it receives $\hat{\theta}$.
}

Note that $G$ and $C$, are fixed, while $S E, C E$, and $K$ are left for design. Both decoders are functions of the encoding schemes and are fixed once $S E$ and $C E$ are determined.

Before constructing a performance metric, we define two parameters that depend on the source encoding scheme and the set $\mathcal{C}_{r}$.

1. $\beta_{\max }\left(\mathcal{C}_{r}\right)=\max _{i, j \in\{1,2, \ldots, L\}}\left\|r_{j}-r_{i}\right\|_{p}$, (diameter of $\mathcal{C}_{r}$ )

2. $\beta_{\min }\left(\mathcal{C}_{r}, S E\right)=\max _{i \in\{1,2, \ldots, M\}} \max _{(k, j) \in \mathcal{B}(i)} \| r_{k}-$ $r_{j} \|_{p}$,

where $\{\mathcal{B}(1), \mathcal{B}(2), \ldots, \mathcal{B}(M)\}$ is a covering of $\mathcal{C}_{r}$, defined by $S E$, that satisfies the following properties:

- $\cup_{i=1}^{M} \mathcal{B}(i)=\mathcal{C}_{r}$,

- $\mathcal{B}(i) \cap \mathcal{B}(j)=\phi$ for all $i \neq j, i, j \in\{1,2, \ldots, M\}$.

See Figure 4 for an illustration of a covering of $\mathcal{C}_{r}$, defined by a source encoder, and the corresponding $\beta_{\max }$ and $\beta_{\text {min }}$.

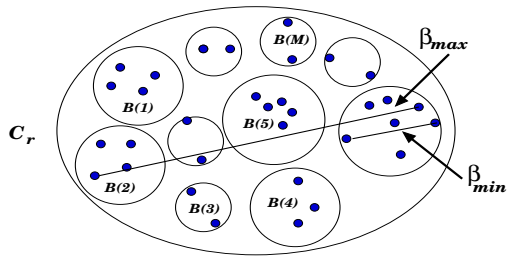

Fig. 4. Source Encoder Compression and $\beta \mathrm{s}$

If the channel is ideal with no noise, then source coding is not necessary, which is equivalent to $M=L$ and $S E=$ $I$. In this case, $\beta_{\min }=0$. If, on the other hand, the source encoder compresses all $L$ signals into 1 "ball" or cover, i.e., $\mathrm{M}=1$, then $\beta_{\min }=\beta_{\max }$. In general, $\beta_{\min }\left(\mathcal{C}_{r}, S E\right)$ is a function that monotonically decreases as $M$ increases, and its shape depends entirely on the source encoder compression algorithm and the set of signals, $r_{k}$, for $k=1,2, \ldots, L$. See Figure 5 for an example.

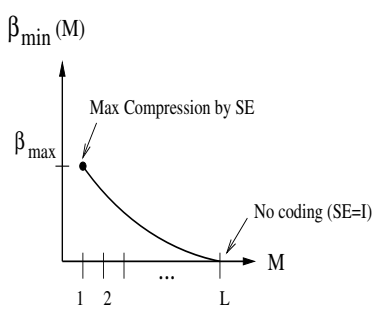

Fig. 5. $\beta_{\min }$ vs. $M$

Going forward, we suppress $\beta_{\text {min }}$ 's dependence on $\mathcal{C}_{r}$, and $S E$ and $\beta_{\text {max }}$ 's dependence on $\mathcal{C}_{r}$, for an easier read. 


\subsection{Model Matching Performance Metric}

In classical synthesis problems, we may be interested in designing $K$ such that $H=(I+G K)^{-1} G K$ is "close" to some given ideal model transfer function $T$. That is, we solve the following problem:

$$
\begin{gathered}
\min _{K}\|H-T\|_{p-i n d} \\
\text { s.t. } H \text { is stable. }
\end{gathered}
$$

Here, we consider the following modified problem that takes into account the communication link in our set up.

$$
\begin{gathered}
\min _{K} \max _{r \in \mathcal{C}_{r}} E\left\{\|H \hat{r}-T r\|_{p}\right\} \\
\text { s.t. } H \text { is stable. }
\end{gathered}
$$

If we let $\alpha=\log _{2}(M)+N$ and $H_{\alpha}=z^{-\alpha} H$ (a delayed version of $H$ ), we then get that

$$
\begin{aligned}
d\left(\hat{y}, y_{\text {ideal }}\right) & \triangleq\|H \hat{r}-T r\|_{p} \\
& =\left\|H_{\alpha}(r+\nu)-T r\right\|_{p} \\
& =\left\|\left(H_{\alpha}-T\right) r+H_{\alpha} \nu\right\|_{p}
\end{aligned}
$$

where $\nu$ is the error signal between $r$ and $\hat{r}$. Basically, the communication link adds a delay of $\alpha$ due to the source and channel encoder schemes, and a distortion, $\nu$, due to the noise induced by the channel. lows:

We now define an indicator random variable, $I_{\theta}$, as fol-

$$
I_{\theta}= \begin{cases}1 & \text { if } \theta=\hat{\theta} \\ 0 & \text { if } \theta \neq \hat{\theta}\end{cases}
$$

Next, we take the expectation of the distance function $d$ with respect to $I_{\theta}$, and get

$$
\begin{aligned}
E_{I_{\theta}}(d)= & P(\theta=\hat{\theta})\left\|\left(H_{\alpha}-T\right) r+H_{\alpha} \nu_{I_{\theta}=1}\right\|+ \\
& P(\theta \neq \hat{\theta})\left\|\left(H_{\alpha}-T\right) r+H_{\alpha} \nu_{I_{\theta}=0}\right\| \\
\leq & P(\theta=\hat{\theta})\left\{\left\|\left(H_{\alpha}-T\right) r\right\|+\left\|H_{\alpha}\right\| \beta_{\min }\right\}+ \\
& P(\theta \neq \hat{\theta})\left\{\left\|\left(H_{\alpha}-T\right) r\right\|+\left\|H_{\alpha}\right\| \beta_{\max }\right\} \\
\leq & \left\|H_{\alpha}\right\|\left\{\beta_{\min }+\left(\beta_{\max }-\beta_{\min }\right) P(\theta \neq \hat{\theta})\right\}+ \\
& \left\|\left(H_{\alpha}-T\right)\right\| \bar{r},
\end{aligned}
$$

where $\bar{r}=\max _{i=1, \ldots, L}|| r_{i} \|_{p}$. From Information Theory [8], we recall that an upper bound on the probability of decoding error, $P(\theta \neq \hat{\theta})$, given any discrete memoryless channel is

$P(\theta \neq \hat{\theta}) \leq(M-1)^{\rho}\left\{\sum_{l=1}^{j}\left[\sum_{m=1}^{k} F(m) p(l \mid m)^{\frac{1}{1+\rho}}\right]^{1+\rho}\right\}^{N}$

$\triangleq \bar{\omega}$,

where $F(m)$ is an arbitrary probability density function on $X$ (typically taken as a uniform density function) and $0 \leq$ $\rho \leq 1$. Finally, we plug in the upper bound, $\bar{\omega}$, into inequality (1) and get

$$
\begin{aligned}
& \qquad E_{I_{\theta}}(d) \leq\left\|\left(H_{\alpha}-T\right)\right\| \cdot \bar{r}+\left\|H_{\alpha}\right\| \eta, \\
& \text { where } \eta \triangleq\left\{\beta_{\min }+\left(\beta_{\max }-\beta_{\min }\right) \bar{\omega}\right\} .
\end{aligned}
$$

\subsection{Tradeoffs Between Communication and Control Ob- jectives}

We now make some high-level observations on components of the upper-bound of $E_{I_{\theta}}(d)$ computed above as the code lengths vary.

- $\left\|\left(H_{\alpha}-T\right)\right\| \cdot \bar{r}$ : increases if $N$ or $M$ increases.

- $\left\|H_{\alpha}\right\| \eta$ : generally increases if $M$ increases, and decreases if $N$ increases.

Overall, if $M$ is fixed and $N$ increases, the estimate of the reference signal improves $\left(\bar{w}\right.$ decreases ${ }^{2}$ ), but the delay of the control system receiving $\hat{r}$ increases, which negatively impacts performance. If $M$ increases and $N$ is fixed, the source encoder more accurately represents the input signals (less compression), but the probability of decoding error increases as there are more possible messages that can be sent through the channel. In addition, delay of the control system receiving $\hat{r}$ again increases, which negatively impacts performance. We set out to quantify these tradeoffs.

\subsection{Problem Statement}

In this section, we state questions that we are interested in answering for the above set up. We assume that the reference signals in $\mathcal{C}_{r}$ lie in $l_{2}$, and the output signals lie in $l_{\infty}$. Thus, the induced norm between the input, $r \in \mathcal{C}_{r}$, and output, $\hat{y}$, is upper-bounded by the $\mathcal{H}_{2}$ norm of the network.

We observe that

$$
\begin{array}{r}
E_{I_{\theta}}(d) \leq\left\|\left(H_{\alpha}-T\right)\right\|_{\mathcal{H}_{2}} \cdot \bar{r}+\left\|H_{\alpha}\right\|_{\mathcal{H}_{2}} \cdot \eta \\
\leq \sqrt{2} \sqrt{\left\|\left(H_{\alpha}-T\right)\right\|_{\mathcal{H}_{2}}^{2} \cdot \bar{r}^{2}+\left\|H_{\alpha}\right\|_{\mathcal{H}_{2}}^{2} \cdot \eta^{2}} \\
\quad=\sqrt{2}\left\|\left[\begin{array}{ll}
\left(H_{\alpha}-T\right) \bar{r} & H_{\alpha} \eta
\end{array}\right]\right\|_{\mathcal{H}_{2}} .
\end{array}
$$

To get the 2nd inequality above, we let $\left|g_{1}\right| \triangleq \|\left(H_{\alpha}-\right.$ $T)\left.\right|_{\mathcal{H}_{2}} \cdot \bar{r},\left|g_{2}\right| \triangleq\left\|H_{\alpha}\right\|_{\mathcal{H}_{2}} \cdot \eta$, and then use the fact that $\left|g_{1}\right|+\left|g_{2}\right| \leq \sqrt{2} \sqrt{\left|g_{1}\right|^{2}+\left|g_{2}\right|^{2}}$.

Now, instead of solving (*), which, in general, is not easily computable for broad classes of encoders and channels, we seek to minimize the above upper bound by solving the following problem.

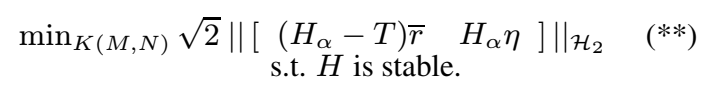

Note that if the channel is ideal $(\bar{w}=0)$, then no coding is necessary, which makes $\beta_{\min }=0$, and therefore $\eta=$ 0 . The above cost function then reduces to the traditional model matching cost function.

\footnotetext{
${ }^{2}$ We note that $\bar{w}$ decreases as $N$ increases only if the channel encoder rate, $\frac{\log _{2}(M)}{N}$, is less than the Shannon Capacity of the Channel, $C$ [8]. The channel encoder rate is defined as the number of input symbols entering $C E$ divided by the number of output symbols leaving $C E$.
} 


\subsubsection{Questions of Interest}

Given a causal, unstable, DT, SISO plant, $G$, a causal, stable, DT, SISO ideal model, $T$, a discrete memoryless channel, $C$, and a decreasing function $\beta_{\min }(M)$,

1. Solve $(* *)$ to synthesize a SISO LTI controller, $K^{o}$, as a function of $(M, N)$.

2. Plug $K^{o}(M, N)$ back into the performance metric and find the code lengths, $M$ and $N$, that minimize the cost function.

3. Describe the sensitivity of the optimal cost to the poles of the plant and ideal model, and to the channel noise.

\section{FIRST-ORDER EXAMPLE}

In this section, we consider the special case where:

- $G(z)=\frac{z}{z-a} \quad|a|>1$,

- $T(z)=\frac{z}{z-\lambda} \quad|\lambda|<1$,

- $C$ is a binary symmetric channel (BSC), with bit-flip probability $p$,

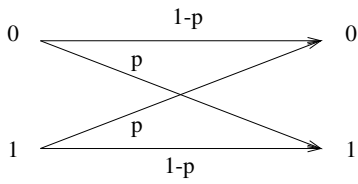

- $\beta_{\max }=1$,

- $\beta_{\text {min }}(M)=\left\{\begin{array}{cc}1 & M=1 \\ \frac{1}{\log _{2}(M)} & M \geq 2 .\end{array}\right.$

\subsection{Synthesis of Controller}

To synthesize the controller as a function of the code lengths, we first parameterize the set of all stabilizing controllers of the remote system $H=(I+G K)^{-1} G K$ [6]. To do so, we first construct one observer-based controller by finding scalars $f$ and $l$ such that $a+a f$ and $a+l$ are both stable (have magnitude inside the unit disk). We choose $f=-1$ and $l=-a$. Then, using the method and notation described in [6], we get the following coprime factorization over all stable proper rational functions of the plant, $G$ :

$$
N(z)=1 \quad M(z)=\frac{z-a}{z} \quad \tilde{Y}(z)=1 \quad \tilde{X}(z)=\frac{a}{z},
$$

where

$$
G(z)=\frac{N(z)}{M(z)} \quad \& \quad N(z) \tilde{X}(z)+M(z) \tilde{Y}(z)=1 .
$$

Then, the set of all stabilizing controllers are of the form $\frac{\tilde{X}(z)+M(z) Q(z)}{\tilde{Y}(z)-N(z) Q(z)}$, for $Q(z)$ being any proper rational stable function. This gives us the following closed-loop transfer function,

$$
\begin{aligned}
H(z) & =N(z) \tilde{X}(z)+N(z) M(z) Q(z) \\
& =P(z)-U(z) Q(z),
\end{aligned}
$$

where

$$
P(z) \triangleq N(z) \tilde{X}(z), \quad U(z) \triangleq-N(z) M(z) .
$$

The optimization problem $(* *)$, using the parameterization of all stabilizing controllers, is then equivalent to solving

$$
\min _{Q(M, N)} \sqrt{2}|| \bar{G}-S \|_{\mathcal{H}_{2}},
$$

s.t. $\{S \in(\bar{u} Q) \bar{V} \mid Q$ is a stable proper rational function $\}$,

where

$$
\begin{gathered}
\bar{G}=\left[\begin{array}{cc}
\bar{r}\left(z^{-\alpha} P-T\right) \quad \eta z^{-\alpha} P
\end{array}\right] \\
\bar{u}=z^{-\alpha} U \\
\bar{V}=\left[\begin{array}{ll}
\bar{r} & \eta
\end{array}\right] .
\end{gathered}
$$

Note that we supress the $\mathrm{z}$-dependence on $\mathrm{z}$-transforms for a more compact notation (eg. $U=U(z)$ ).

Before solving for the optimal $S^{o}$, we recall that any stable proper rational function can be written as the product of an all-pass filter and a minimum-phase filter (see [6] for details). We can then factor $\bar{u}$ as follows:

$$
\bar{u}=\bar{u}_{a p} \bar{u}_{m p}=\left\{\frac{a-z}{\left(a-z^{-1}\right)\left(z^{\alpha+1}\right)}\right\}\left\{a-z^{-1}\right\} .
$$

Finally, we define $S^{o}=\bar{u} Q^{o} \bar{V}$, and for all stable rational proper functions $Q$, the following statements hold (in $\mathcal{H}_{2}$ norm).

$$
\begin{aligned}
\|\bar{G}-S\| & =\|\bar{G}-\bar{u} Q \bar{V}\| \\
& =\left\|\bar{G}-\bar{u}_{a p} \bar{u}_{m p} Q \bar{V}\right\| \\
& =\left\|\bar{u}_{a p}\left(\bar{u}_{a p}^{-1} \bar{G}-\bar{u}_{m p} Q \bar{V}\right)\right\| \\
& =\left\|\bar{u}_{a p}^{-1} \bar{G}-\bar{u}_{m p} Q \bar{V}\right\| \\
& =\left\|\left[\bar{u}_{a p}^{-1} \bar{G}\right]_{\mathcal{H}_{2}}+\left[\bar{u}_{a p}^{-1} \bar{G}\right]_{\mathcal{H}_{2}^{\perp}}-\bar{u}_{m p} Q \bar{V}\right\| \\
& =\left\|\left[\bar{u}_{a p}^{-1} \bar{G}\right]_{\mathcal{H}}{ }_{\frac{1}{2}}\right\|+\left\|\left[\bar{u}_{a p}^{-1} \bar{G}\right]_{\mathcal{H}_{2}}-\bar{u}_{m p} Q \bar{V}\right\| .
\end{aligned}
$$

From the last equality it is easy to see that $\bar{u}_{m p} Q^{o} \bar{V}=$ $\left[\bar{u}_{a p}^{-1} \bar{G}\right]_{\mathcal{H}_{2}}$. The optimal parameter function is then

$$
Q^{o}=\frac{\left(\bar{u}_{m p}\right)^{-1}}{\overline{V V}^{*}}\left[\bar{u}_{a p}^{-1} \bar{G}\right]_{\mathcal{H}_{2}} \bar{V}^{*} .
$$

Recall that $[f]_{\mathcal{H}_{2}}$ is the projection of a function $f$ onto the $\mathcal{H}_{2}$ subspace, and $g^{*}$ denotes the complex-conjugate transpose of a complex-valued vector function $g$.

Note that $\bar{u}$ and $\bar{V}$ are functions of $M$ and $N$, therefore, $Q^{o}$ is also a function of $M$ and $N$. Finally, the optimal controller, $K^{o}=\frac{Q^{\circ}}{1-G Q^{\circ}}$, is a function of $M$ and $N$. For our first-order example, 


$$
H^{o}(M, N)=\frac{C 1 z}{a z-1}+\frac{C 2 z}{z-\lambda}
$$

where

$$
\begin{gathered}
C_{1}=\left(a-\frac{1}{a}\right)\left[\frac{a \gamma}{k(a \lambda-1)}+1\right], \\
C_{2}=\frac{\gamma(\lambda-a)}{k(a \lambda-1)}, \\
k=\bar{r}^{2}+\eta^{2}, \\
\gamma=\lambda^{\alpha}\left[\frac{\left(a^{2}-1\right) \bar{r}^{2}}{\lambda-a}+a \bar{r}^{2}\right] .
\end{gathered}
$$

\subsection{Synthesis of Code Lengths}

Now that we have the optimal closed-loop transfer function as a function of code lengths, we look for the optimal $\left(M^{o}, N^{o}\right)$ pair that minimizes the cost function $\sqrt{2} \| \bar{G}-$ $S \|_{\mathcal{H}_{2}}$, and the corresponding optimal closed-loop transfer function $H^{o}$. We set $a=1.2, \lambda=0.95, p=0.01$, and $\bar{r}=0.2$ and find that the minimum cost is 0.6194 and occurs when $M^{o}=32$, and $N^{o}=13$. The corresponding optimal control system is

$$
H^{o}=\frac{0.0687 z}{1.2 z-1}+\frac{0.1693 z}{z-0.95} .
$$

\subsection{Performance Sensitivity}

In this section, we investigate the sensitivity of the optimal cost to the poles of the plant and model, and to the channel noise.

Figure 6 illustrates how the channel code length, $N$, impacts the optimal cost for different unstable plant poles, when $\lambda=0.95$ and the bit-flip probabilities of the BSC are $0.01,0.2$, and 0.4 . Figure 7 illustrates the optimal cost for different levels of channel noise when $\lambda=0.12$. For these experiments, $\bar{r}=0.2$, and $M=1$, while $N$ and $a$ varied. tions.

From Figures 6 and 7, we make the following observa-

- Sensitivity to plant pole: The optimal code length increases as the magnitude of the unstable pole increases.

- Sensitivity to channel noise: As the channel noise increases, more coding is necessary to reach a minimum cost. However, for very noisy channels, the optimal code length is too long to be of use when implemented, as the delay is too large. Essentially, one should not code when the channel is too noisy.

- Sensitivity to ideal model pole: The closer the model pole is to the unit disk, the more coding improves performance. That is, we see the tradeoffs between sending the remote system an accurate reference command and meeting performance.

\subsection{Ideal Solution}

In this section, we look at performance sensitivity of the ideal model matching problem (no channel or coding) by setting $\eta=\alpha=0$. Figure 8 illustrates how the optimal cost behaves as the plant pole becomes more unstable (as $a$ ranges from 1.01 to 4.1 ) for different ideal model poles
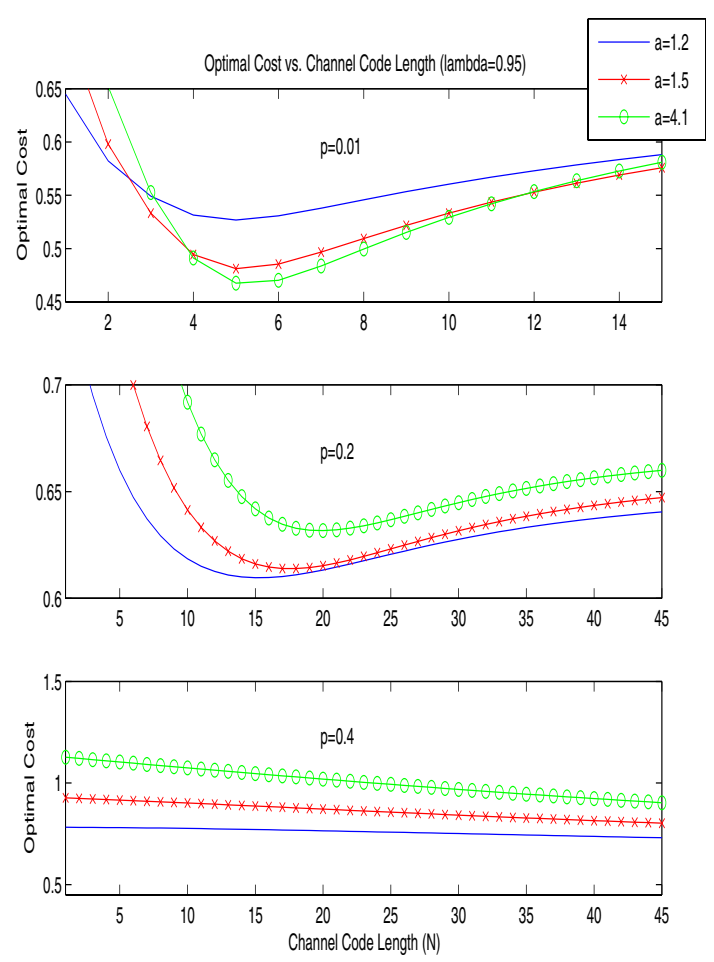

Fig. 6. Optimal Cost vs. Channel Code Length $(N):(\lambda=$ $0.95)$

( $\lambda=0.95$ and 0.02). As shown in Figure 8 , we see that when the ideal model pole is close to the unit disk, the ideal optimal cost $\left(|| H-T \|_{\mathcal{H}_{2}}\right)$ is lowest when $a=4.1$. This is consistent with what we see in Figure 6 for very low channel noise $(p=0.01)$, which shows that the optimal cost for $a=4.1$ is lowest. However, when the ideal model pole is close to the origin, the ideal optimal cost is lowest when $a=1.01$, which is also consistent with what we see in Figure 7 for very low channel noise.

\section{CONCLUSIONS AND FUTURE WORK}

In this paper, we simultaneously synthesized the controller and encoder block lengths that meet specified model matching objectives for a first-order plant and model case. We also illustrated performance sensitivity to the poles of the plant and ideal model, and to the channel noise. In short, the tradeoffs between sending an accurate reference command (by implementing some channel coding) and matching an ideal model are most prominent when the channel is not too noisy, the plant pole is more unstable, and when the dynamics of the ideal model are "slow" (ideal model pole is close to the unit disk).

Future work entails making the set up more "real-time" and appropriate for navigation of the remote system. In this paper, only one reference signal is sent through the link, and the system waits $\alpha$ time steps before it receives any signal. A more realistic set up includes switching the reference signal over time, and allowing for $\hat{r}$ to reach the system without much delay, and to have it improve (be closer to $r$ ) over 

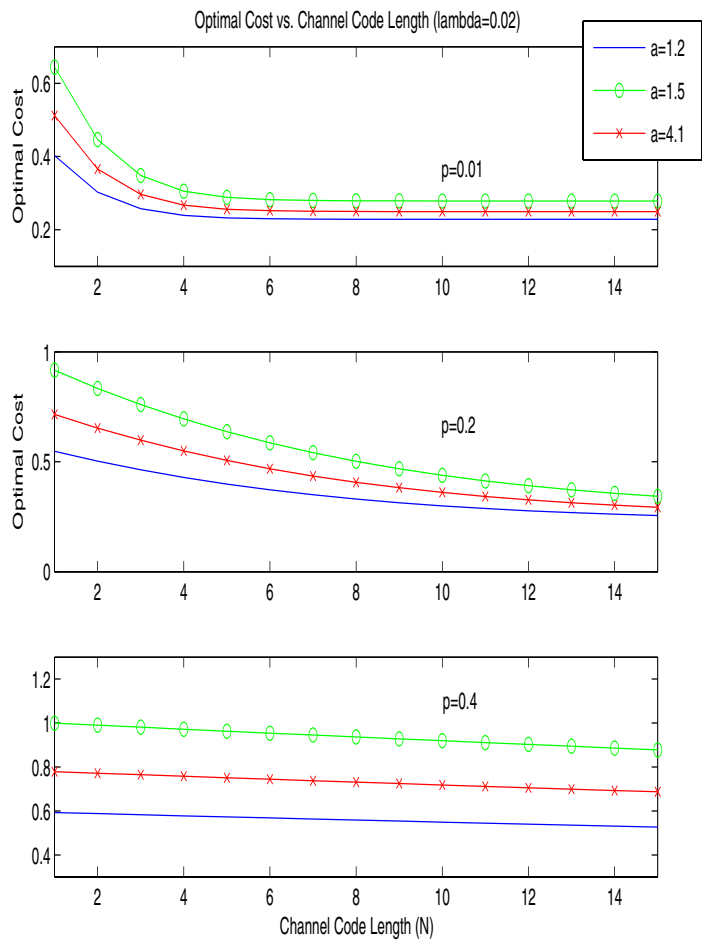

Fig. 7. Optimal Cost vs. Channel Code Length $(N):(\lambda=$ $0.02)$
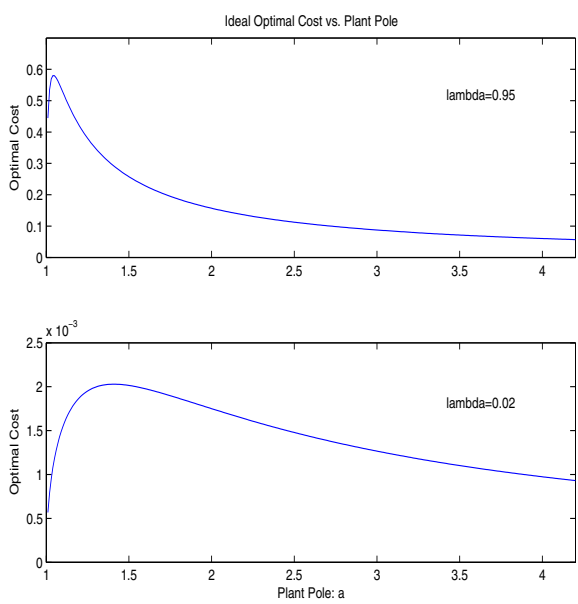

Fig. 8. Ideal Optimal Cost vs. Plant Pole

time as the input gets further coded. The latter scheme entails implementing a dynamic encoding scheme, much like that introduced in [15]. We also would like to explore different classes of encoders, such as convolutional or interleaver encoders [8].

\section{REFERENCES}

[1] Baillieul, J., "Feedback Coding for Information-based Control: Operating Near the Data-Rate Limit," Proceedings of the 41st IEEE Conference on Decision and Control, December 2002.

[2] Borkar, V.S., Mitter, S., "LQG Control With Communication Constraints," Communications, Computation, Control and Signal Proceedings, Chapter 21, 1997.

[3] Brockett, Roger W., "Quantized Feedback Stabilization of Linear Systems,"IEEE Transactions on Automatic Control: vol. 45, pp. 1279-1289, 2000.

[4] Wong, Wing Shing, Brockett, Roger, "Systems with Finite Communication Bandwidth Constraints-II: Stabilization with Limited Information Feedback," IEEE Transactions on Automatic Control: vol. 44, no. 5, May 1999.

[5] Delchamps, David, "Stabilizing a Linear System with Quantized State Feedback," IEEE Transactions on Automatic Control: vol. 35, no. 8, August 1990.

[6] Doyle, John, Francis, Bruce, Tannenbaum, Allen "Feedback Control Theory," Macmillan Publishing Company; New York, c1992.

[7] Elia, Nicola, Mitter, Sanjoy "Stabilization of Linear Systems With Limited Information," IEEE Transactions on Automatic Control: vol. 46, no. 9, September 2001.

[8] Gallager, Robert, "Information Theory and Reliable Communication," John Wiley and Sons, Inc.; New York, c1968.

[9] Liberzon, Daniel, "A note on stabilization of linear systems using coding and limited communication," Proceedings of the 41st IEEE Conference on Decision and Control, December 2002.

[10] Kitts, C. "Development and Teleoperation of Robotic Vehicles," AIAA Unmanned Unlimited Systems, Technologies and Operations Conference, San Diego, California, 2003.

[11] Martins, N.C. and Dahleh, M. A., "Fundamental Limitations of Disturbance Attenuation in the Presence of Finite Capacity Feedback, American Control Conference 2005 (Extended version submitted to the IEEE Transactions in Automatic Control).

[12] Martins, N.C., Dahleh M.A., and Elia N.; Stabilization of Uncertain Systems in the Presence of a Stochastic Digital Link, IEEE CDC, 2004 (Extended version submitted to the IEEE Transactions in Automatic Control).

[13] Nair, G.N., Evans, R.J., "Stabilization with Data-rate-limited Feedback: tightest attainable bounds," Systems and Control Letters, volume 41, 2000, pp. 49-56.

[14] Nair, G.N., Evans, R.J. "Stabilizability of stochastic linear systems with finite feedback data rates", SIAM Journal on Control and Optimization, Society for Industrial and Applied Mathematics, USA, vol. 43, no. 2, pp. 413 - 436, July 2, 2004.

[15] Sarma, Sridevi, Dahleh, Munther A., Salapaka, Srinivasa, "On Time-Varying Bit-Allocation Maintaining Stability: A Convex Parameterization," Proceedings of the 43rd IEEE Conference on Decision and Control, December 2004.

[16] Tatikonda, Sekhar, "Control Under Communication Constraints," IEEE Transactions on Automatic Control : Volume 49, Issue 7, July 2004 Page(s): 1056 - 1068.

[17] Yuksel, Serder, Basar, Tamer, "State Estimation and Control for Linear Systems over Communication Networks," Proceedings of 2003 IEEE Conference on Control Applications, Volume 1, June 2003.

[18] P.G. Voulgaris, C. Hadjicostis, R. Touri, "A perfect reconstruction paradigm for digital communications," Proceedings of the 42nd IEEE Conference on Decision and Control, Dec. 2003. 